\title{
Cranial Bone Regeneration by Controlled Release of Platelet Growth Factors from Biodegradable Hydrogel
}

\author{
Akishige Hokugo ${ }^{1)}$, Yasunori Sawada ${ }^{2)}$, Kozo Mushimoto²), Shosuke Morita ${ }^{2)}$ and Yasuhiko Tabata ${ }^{1)}$ \\ 1) Department of Biomaterials, Field of Tissue Engineering, Institute for Frontier Medical Sciences, Kyoto University, Kyoto, Japan \\ 2) First Department of Oral and Maxillofacial Surgery, Osaka Dental University, Osaka, Japan
}

\begin{abstract}
Recently, platelet-rich plasma (PRP) has been clinically employed to promote bone repairing. However, little has been investigated on the materials combination of PRP to enhance the biological function of growth factors present in platelets. In this study, feasibility of gelatin hydrogels in the controlled release of platelet growth factors and the consequent enhancement of PRP-induced bone regeneration were evaluated by a cranial bone defect model of rabbits. In conclusion, the gelatin hydrogel achieved the controlled release of bioactive platelet growth factors to significantly promote bone regeneration, in marked contrast to PRP alone.
\end{abstract}

Key words: Bone regeneration, Drug Delivery system (DDS), Platelet-rich Plasma (PRP), Gelatin hydrogel

\section{Introduction}

One of the currently great interests in the surgery of oral and maxillofacial bone grafting is to enhance bone formation and to increase the rate of bone graft healing by use of platelet-rich plasma (PRP). Aghaloo et $\mathrm{al}^{1)}$ have evaluated the effect of PRP on cranial bone healing, but no significant enhancement of bone formation, was seen radiographically or histomorphometrically. On the contrary, some researches have demonstrated the activity of PRP combined with biomaterials to promote the bone regeneration ${ }^{2}$. However, it is rather questionable whether or not combination with biomaterials allows PRP to enhance the biological activity ${ }^{3)}$. There is no clear scientific explanation for the material role in the PRP-induced augmentation of bone repairing ${ }^{4)}$. Little has been investigated on the biological function of PRP from the viewpoint of combination materials.

The objective of the present study is to investigate potentiality of gelatin hydrogel as the combination materials of PRP. Gelatin is a biodegradable material which has been extensively utilized for pharmaceutical and medical purposes, and proven to be biosafe through its long clinical applications ${ }^{5}$. We have prepared hydrogels from gelatin and succeeded in the controlled release of bioactive bFGF, transforming growth factor-â (TGF- $\beta 1$ ), platelet-derived growth factor (PDGF), and bone morphogenetic protein 2 (BMP2) to enhance their biological functions ${ }^{6}$. It is well recognized that the secretory a granules of platelets and contain various growth factors, such as PDGF, TGF- $\beta$ ). It is highly expected that the gelatin hydrogel functions as a release carrier of the platelet growth factors. Following the PRP was incorporated into the hydrogel and applied to the cranial defect of rabbit bone, PRP-induced promotion of bone regeneration was evaluated.

Preparation of PRP

\section{Materials and Methods}

New Zealand White rabbits (fifteen) ranging from 3.0 to 3.5 kg of body weight (Shimizu Laboratory Animal Supply Co. Ltd., Kyoto, Japan) were used. All the animal experiments have been

Correspondence to Yasuhiko Tabata, Ph.D., D.Med.Sci., D. Pharm.

Department of Biomaterials, Field of Tissue Engineering, Institute for Frontier Medical Sciences, Kyoto University, 53 Kawara-cho Shogoin, Sakyo-ku, Kyoto 606-8507, JapanTel:81-75-751-4121 Fax:81-75-7514646e-mail: yasuhiko@frontier.kyoto-u.ac.jp approved by the Kyoto University Committee for Animal Experimentation. PRP was prepared according to the method reported $^{1)}$. Approximately volume of PRP obtained was $0.8 \mathrm{ml}$. The density of platelets in the PRP prepared increased by 5.91 times as much as that of original peripheral blood.

\section{Preparation of gelatin hydrogel incorporating $P R P$}

Gelatin hydrogels were prepared by glutaraldehyde crosslinking of gelatin as reported previously ${ }^{8}$. The water content of gelatin hydrogel (the weight ratio of water present in the hydrogel to the wet hydrogel) was $98 \mathrm{wt} \%$. The hydrogel freeze-dried was cut into a disk shape (5 mm in diameter and $3 \mathrm{~mm}$ height) and then sterilized with ethylene oxide gas. PRP $(100 \mathrm{ml})$ prepared was dropped onto the freeze-dried gelatin hydrogel disk, followed by leaving for $1 \mathrm{hr}$ at $37^{\circ} \mathrm{C}$ for PRP impregnation to obtain the hydrogel incorporating PRP.

\section{In vivo experiment}

In vivo experiment was performed by the surgical procedure previously reported with modification ${ }^{1}$. An incision was made to the bony cranium and the periosteum was reflected. Bone defects of 5-mm diameter were carefully created with a trephine bur and the four defects were randomly applied with a gelatin hydrogel incorporating PRP, PRP (100 ml) activated with $10 \mathrm{ml}$ of bovine thrombin (Jones, St.Louis, MO) (1000 IU), and an empty gelatin hydrogel, or not applied as a control (Figure 1). The wound was closed with sutures and a postoperative antibiotic (Fosmicin ${ }^{\circledR}$, Meiji Seika, Tokyo, Japan) was administered intramuscularly at a dose of $100 \mathrm{mg} / \mathrm{kg}$ per a day for 3 days.

\section{Assessment of Bone regeneration}

Bone regeneration at the site of bone defect was assessed using soft x-ray, peripheral Quantitative Computed Tomography (pQCT), and histological examinations 8 weeks after application. Soft x-ray photographs of bone specimen were taken on Soft xray (Softex ${ }^{\circledR}$ CBM-2, Tokyo, Japan). The bone mineral density (BMD) of each bone defect was measured using pQCT ( Stratec XCT 960M, Norland Medical Systems, USA). Bone specimens were placed into $10 \%$ neutral phosphate-buffered formaldehyde, decalcified with $10 \%$ formic acid, and processed for the paraffin embedding. Sections of $3 \mathrm{~mm}$ thickness were prepared and stained 
with hematoxylin and eosin to view by a light microscopy (AX80T, Olympus, Tokyo, Japan).

\section{Statistical Analysis}

All the data were statically analyzed using Fisher's least significant difference test for multiple comparisons and statistical significance was accepted to be less than 0.05 . The experimental results were expressed as the mean \pm the standard deviation of the mean.

\section{Results}

Figure 2 shows soft $\mathrm{x}$-ray photographs of bone defects 8 weeks after application with the gelatin hydrogel incorporating PRP or other agents. When the bone defect was applied with the gelatin hydrogel incorporating PRP (Figure 2a) and the PRP-activated thrombin (Figure 2b), bone regeneration at the defect was radiographically detected although the extent of radiopaque area for the former was larger than that of the latter. On the other hand, The radiopaque was observed for the empty gelatin hydrogel, but there was no radiographical image of bone connection (Figure 2c). No radiographical bone formation was observed at the control defect applied without any application (Figure 2d).

Figure 3 shows histological sections of ulna defects 8 weeks after application. When applied with the gelatin hydrogel incorporating PRP, the bone defect was histologically closed by bone tissue newly regenerated (Figure 3a). Bone regeneration at the defect applied with the PRP-activated thrombin was also observed although the area of bone tissue newly regenerated was smaller (Figure 3b). On the contrast, less bone regeneration was observed for the empty gelatin hydrogel application and there was a gap area between bones newly regenerated in the central area of bone defect (Figure 3c). No bone regeneration was detected at the defect not applied, while remarkable ingrowth of soft connective tissue into the defect was observed (Figures 3d).

Figure 4 shows the BMD values at cranial bone defects 8 weeks after application with the gelatin hydrogel incorporating PRP or other agents. The BMD value at the bone defect applied with the gelatin hydrogel incorporating PRP and the PRP-activated thrombin was significantly higher than that of the empty gelatin hydrogel and no application.

\section{Discussion}

It has been recognized that platelets present in the PRP secrete several growth factors accompanied with the degranulation of a granules and the factors secreted exhibit various biological activities $^{9)}$. It is well known that collagen or thrombin is a substance to trigger the platelet aggregation to secrete platelet growth factors ${ }^{10)}$. In addition, we have demonstrated that gelatin is one of substances to trigger the platelet activation for growth factor secretion $^{11)}$.

In the living tissue, generally various growth factors are stored interacting with extracellular matrix (ECM) components, such as acidic polysaccharides, through various intermolecular interaction forces ${ }^{12)}$. Furthermore, this physicochemical interaction enables the growth factors to maintain and enhance their biological functions in vivo. We have succeeded the controlled release of TGF-b1 from the hydrogel of acidic gelatin to demonstrate the bone regeneration at rabbit and monkey skull defects, in marked contrast to free TGF-b1. It was found that TGF-b1 was sorbed into the acidic gelatin hydrogel mainly due to the electrostatic interaction between the gelatin and TGF-b1 molecules ${ }^{13)}$. Similarly, PDGF is also released from the acidic gelatin hydrogel. As the interaction between PRP and the gelatin hydrogel, it is likely that the platelets of PRP is activated by exposure to gelatin molecules during the impregnation process, resulting in the secretion of

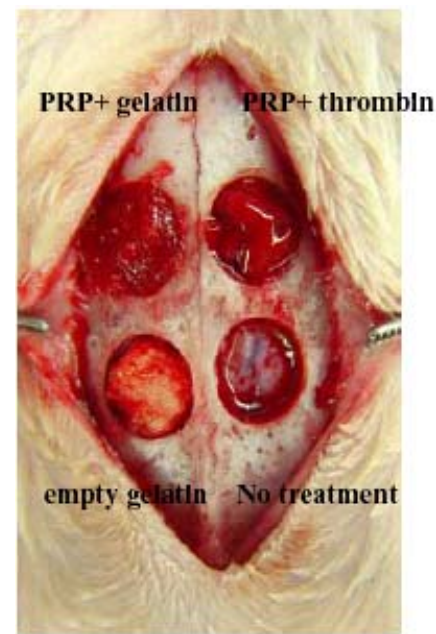

Fig. 1. A photograph of rabbit cranial bone with surgical sites applied.
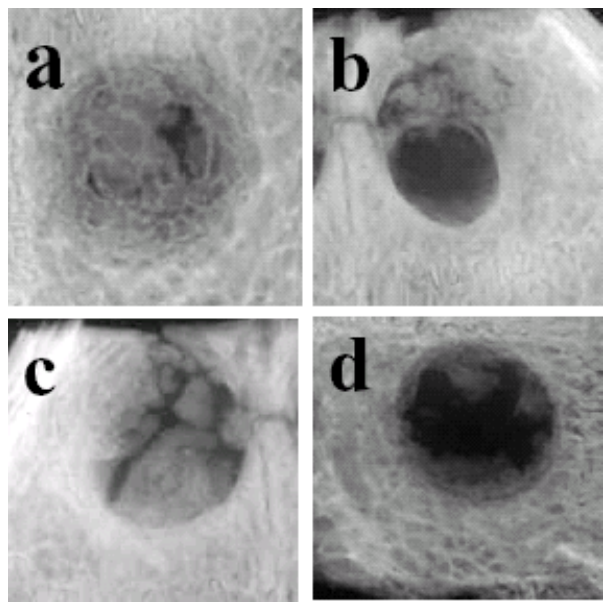

Fig. 2. Radiographic pictures of cranial bone defects 8 weeks after application with a gelatin hydrogel incorporating PRP (a), PRPactivated thrombin (b), and an empty gelatin hydrogel (c) or without any application (d).
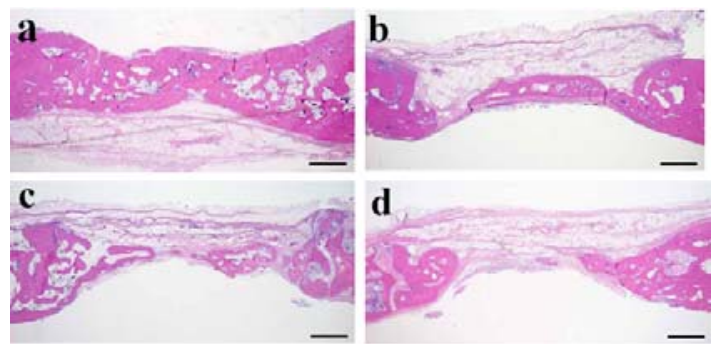

Fig. 3. Histological sections of cranial bone defects 8 weeks after application with a gelatin hydrogel incorporating PRP (a), PRP-activated thrombin (b), and empty gelatin hydrogel (c) or without any application (d). The bar length is $1.0 \mathrm{~mm}$

PDGF and TGF-b1 in the hydrogel. The growth factors secreted will be immobilized into the hydrogel through their interaction with gelatin molecules. As a result of hydrogel degradation, the growth factors immobilized are released from the hydrogel which is similar to the case described above ${ }^{6,13)}$. 
International symposium of Maxillofacial \& Oral Regenerative Biology in Okayama 2005

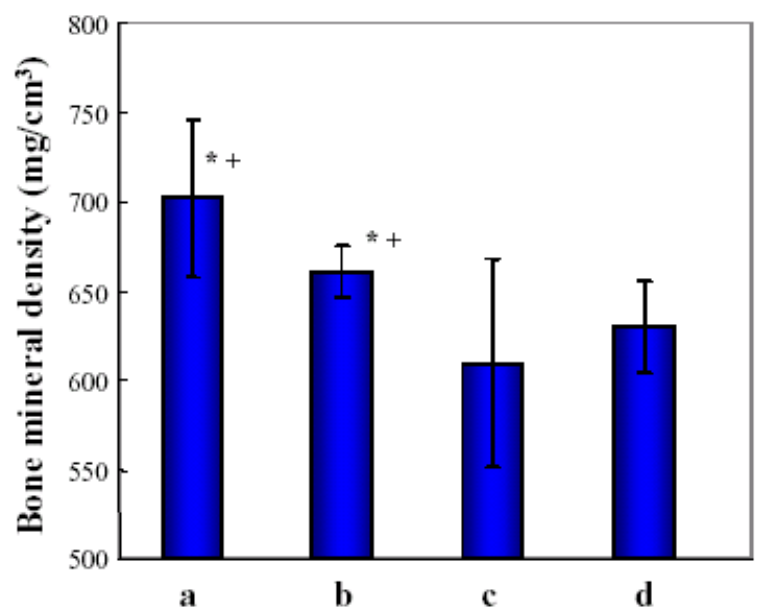

Fig. 4. The BMD values at cranial bone defects 8 weeks after application with gelatin hydrogels incorporating PRP (a), PRP-activated thrombin (b), and empty gelatin hydrogels (c) or without any application (d). *, $\mathrm{P}<0.05$; significance against the BMD value at the bone defect applied with the empty gelatin hydrogels; +, $\mathrm{P}<0.05$; significance against the $\mathrm{BMD}$ value at the bone defect without any application.

A promising scaffold used to deliver osteoinductive growth factors at the bone defect site should not only assist bone regeneration at the defect as a scaffold, but also promote biological effects 14). When the rate of scaffold biodegradation is too fast compared with the rate of bone regeneration at the defect, the scaffold does not physically prevent the soft tissue infiltration, resulting no bone regeneration. In this study, bone regeneration was observed for the PRP-activated with thrombin, but the regenerated bone area was small (Figure $3 \mathrm{~b}$ ). The fibrin clot prepared by PRP activated with thrombin would neither function as a release carrier of platelet growth factors nor a scaffold for bone regeneration. In addition, since the clot was degraded fast, it is possible that it did not efficiently prevent the infiltration of soft tissue into the bone defect and consequently suppressed regeneration of bone tissue. On the contrary, the gelatin hydrogel released the platelet growth factors to effectively promote their osteoinductive activity at the defect, while the hydrogel presence functions to physically prevent the soft tissue infiltration. It is possible that the hydrogel was being harmonized with the process of bone regeneration, resulting in the defect closure by the bone tissue newly formed (Figure 3a). The BMD value of defects applied with the gelatin hydrogel incorporating PRP was significantly higher than that of other groups (Figure 4). This finding indicates that a balance in the time profile between growth factors release and bone regeneration is essential for bone regeneration induced by the growth factors of PRP released. The gelatin hydrogel used in this study possessed a suitable in vivo degradability, which would functions not only as a space maker to prevent in growth of soft tissue into the defect, but also as a release carrier of PRP growth factors.

The gelatin hydrogel is a promising scaffold capable for the controlled release of PRP growth factors to promote bone regeneration.

\section{References}

1. Aghaloo TL, Moy PK, Freymiller EG. Investigation of plateletrich plasma in rabbit cranial defects: A pilot study. J Oral Maxillofac Surg 60:1176-81, 2002

2. Kassolis JD, Rosen PS and Reynolds MA. Alveolar ridge and sinus augmentation utilising platelet-rich plasma in combination with freeze-dried bone allograft: case series. J Periodontol 71:1654, 2000.

3. Rosenberg ES and Torosian J. Sinus grafting using plateletrich plasma: Initial case presentation. Pract Periodontics Aesthet Dent 12:84, 2000.

4. Sanchez AR, Sheridan PJ and Kupp LI. Is platelet-rich plasma the perfect enhancement factor? A current review. Int J Oral Maxillofac Implants 18:93, 2003

5. Zekorn D. Intravascular retention, dispersal, excretion and break-down of gelatin plasma substitutes. Bibl Haematol 33:131, 1969

6. Tabata Y. Tissue regeneration based on growth factor release. Tissue Eng 9Suppl 1:S5, 2003

7. Harrison P and Cramer EM. Platelet alpha granules. Blood Rev 7:52, 1993

8. Ozeki M and Tabata Y. Promoted growth of murine hair follicles through controlled release of basic fibroblast growth factor. Tissue Eng 8:359, 2002

9. Marx RE. Platelet-rich plasma: evidence to support its use. J Oral Maxillofac Surg 62:489, 2004

10. Kaplan KL, Nossel HL, Drillings M and Lesznik G. Radioimmunoassay of platelet factor 4 and betathromboglobulin: development and application to studies of platelet release in relation to fibrinopeptide A generation. $\mathrm{Br}$ J Haematol 39:129, 1978

11. Hokugo A, Ozeki M, Kawakami O, Sugimoto K, Mushimoto K, Morita S, Tabata Y. Augmented bone regeneration activity of platelet-rich plasma by biodegradable gelatin hydrogel. Tissue Eng 11:1224-33, 2005

12. Taipale J and Keski-Oja J. Growth factors in the extracellular matrix. FASEB J 11:51, 1997

13. Yamamoto M, Tabata Y, Hong L, Miyamoto S, Hashimoto N and Ikada Y. Bone regeneration by transforming growth factor $\beta 1$ released from a biodegradable hydrogel. J Controlled Release 64:133, 2000

14. Hong L, Tabata Y, Miyamoto S, Yamamoto M, Yamada K, Hashimoto $\mathrm{N}$ and Ikada Y. Bone regeneration at rabbit skull defects treated with transforming growth factor-beta 1 incorporated into hydrogels with different levels of biodegradability. J Neurosurg 92:315, 2000 